\title{
A CONTRIBUTION TO THE LICHEN FAMILY GRAPHIDACEAE (OSTROPALES, ASCOMYCOTA) OF BOLIVIA. 2
}

\author{
Ulf Schiefelbein, Adam Flakus, Harrie J. M. Sipman, \\ Magdalena Oset \& Martin KuKwa ${ }^{1}$
}

\begin{abstract}
Microlichens of the family Graphidaceae are important components of the lowland and montane tropical forests in Bolivia. In this paper we present new records for 51 taxa of the family in Bolivia. Leiorreuma lyellii (Sm.) Staiger is reported as new for the Southern Hemisphere, while Diploschistes caesioplumbeus (Nyl.) Vain., Graphis daintreensis (A. W. Archer) A. W. Archer, G. duplicatoinspersa Lücking, G. emersa Müll. Arg., G. hossei Vain., G. immersella Müll. Arg. and G. subchrysocarpa Lücking are new for South America. Thirty taxa are reported for the first time from Bolivia. Notes on distribution are provided for most species.
\end{abstract}

Key words: biodiversity, biogeography, lichenized fungi, Neotropics, South America

Ulf Schiefelbein, Blücherstr. 71, D-18055 Rostock, Germany

Adam Flakus, Laboratory of Lichenology, W. Szafer Institute of Botany, Polish Academy of Sciences, Lubicz 46, 31-512 Kraków, Poland

Harrie J. M. Sipman, Botanischer Garten \& Botanisches Museum Berlin Dahlem, Königin-Luise-Strasse 6-8, D-14195 Berlin, Germany

Martin Kukwa \& Magdalena Oset, Department of Plant Taxonomy and Nature Conservation, University of Gdańsk, Wita Stwosza 59,80-308 Gdańsk, Poland; e-mail: dokmak@ug.edu.pl

\section{INTRODUCTION}

Bolivia has the highest ecosystem diversity in South America and the forest communities form a mosaic of vegetation which offers a variety of potential habitats for numerous microlichens (Navarro \& Maldonado 2002; Josse et al. 2003; Ibisch \& Mérida 2004; Navarro \& Ferreira 2007). Thus a large number of such species should be expected to occur in Bolivia; however, current knowledge of this group in Bolivia is very incomplete, and the family Graphidaceae is no exception in this regard. On the basis of material we collected recently during lichenological exploration in Bolivia, here we document some of this hidden diversity.

This paper is the second contribution dealing with the family Graphidaceae in Bolivia (Kukwa et al. 2013).

\footnotetext{
1 Corresponding author
}

\section{Material AND METHODS}

Specimens are deposited at B, GOET, KRAM, LPB, UGDA (acronyms after Thiers 2012) and the private herbaria of A. Flakus and U. Schiefelbein. Secondary lichen metabolites were identified by thin layer chromatography (TLC) according to Orange et al. (2001).

Data on general distribution are presented only for species reported for the first time from Bolivia. New national records are asterisked $\left({ }^{*}\right)$, those new for South America have two asterisks $\left({ }^{* *}\right)$, and those new for the Southern Hemisphere have three $\left(^{* * *}\right)$.

The following abbreviations are used in the "Specimens examined" sections: $A F-\mathrm{A}$. Flakus; $M K-\mathrm{M}$. Kukwa; $O P$ - O. Plata; $P R$ - P. Rodriguez Flakus; RIM - R. I. Meneses; US - U. Schiefelbein; RN - Reserva Nacional; AMBORÓ - Parque Nacional y Área Natural de Manejo Integrado Amboró; APOLOBAMBA - Área Natural de Manejo Integrado Nacional Apolobamba; CARRASCO - Parque Nacional Carrasco; COTAPATA - Parque Nacional y Área Natural de Manejo Integrado Cotapata; KAA-IYA - Parque Nacional y Área Natural 
de Manejo Integrado Kaa-Iya del Gran Chaco; SAJAMA - Parque Nacional Sajama; TARIQUÍA - Reserva Nacional de Flora y Fauna Tariquía (abbreviations of protected areas according to SERNAP Bolivia; http://www. sernap.gob.bo).

\section{RESULTS}

"Diorygma antillarum (Vain.) Nelsen, Lücking \& Rivas Plata

A Neotropical species previously reported from Brazil, Costa Rica, El Salvador, the Lesser Antilles, Panama, Peru, Venezuela and the USA (Florida) (Aptroot et al. 2009; Nelsen et al. 2012).

Specimens examined. BOliVia. Dept. Tarija. Prov. Aniceto Arce, Filo de Sidras, $22^{\circ} 14^{\prime} 50^{\prime \prime} \mathrm{S}, 64^{\circ} 33^{\prime} 28^{\prime \prime} \mathrm{W}$, $1064 \mathrm{~m}$, Tucumano-Boliviano submontane forest, on bark, 22 Nov. 2010. AF $18421 \& 18672$ (KRAM, LPB); Serranía de Propiedad Arnold, $22^{\circ} 13^{\prime} 19^{\prime \prime} \mathrm{S}, 64^{\circ} 33^{\prime} 41^{\prime \prime} \mathrm{W}$, $1309 \mathrm{~m}$, Tucumano-Boliviano montane forest, on bark, 24 Nov. 2010, AF 18708 (KRAM, LPB); Prov. Burnet O'Connor, Lomas de la Soledad, road between Entre Ríos and Chiquiacá, $21^{\circ} 39^{\prime} 38^{\prime \prime} \mathrm{S}, 64^{\circ} 07^{\prime} 31^{\prime \prime} \mathrm{W}$, $1670 \mathrm{~m}$, Tucumano-Boliviano altimontane forest, corticolous, 10 Aug. 2012, MK 11264 (LPB, UGDA); TARIQUÍA, near Salinas, $21^{\circ} 49^{\prime} 15^{\prime \prime} \mathrm{S}, 64^{\circ} 12^{\prime} 44^{\prime \prime} \mathrm{W}$, $1400 \mathrm{~m}$, Tucumano-Boliviano montane forest, corticolous, 10 Aug. 2012, MK 11246 (LPB, UGDA).

\section{Diorygma confluens (Fée) Kalb, Staiger \& Elix}

This species was only recently reported from Bolivia from six localities (Kukwa et al. 2013).

Diorygma confluens is chemically variable (Kalb et al. 2004). The material reported so far from Bolivia contained lichexanthone, stictic and constictic acids (major), and sometimes traces of hypoconstictic, hypostictic, norstictic and cryptostictic acids (Kukwa et al. 2013). In one of the specimens cited below ( $M K$ 7111), lichexanthone, hypoconstictic (major), constictic (submajor) and stictic (trace) acids were detected.

Specimens eXamined. BOLIVIA. Dept. Beni. Prov. Ballivian, Palmar, $14^{\circ} 58^{\prime} 56^{\prime \prime} \mathrm{S}, 67^{\circ} 05^{\prime} 16^{\prime \prime} \mathrm{W}, 250 \mathrm{~m}$, by road, corticolous, 2 Aug. 2008, MK 7111 (LPB, UGDA); DePt. LA PAZ. Prov. Iturralde, between Tumapasa and Mamuque, $300 \mathrm{~m}$, garden, corticolous, 5 Mar. 1987, S. Stab LB-33 (B).
Diorygma epiglaucum (Müll. Arg.) Kalb, Staiger \& Elix

Previously known from three records in Bolivia (Kalb et al. 2004; Flakus 2008).

Specimens examined. BOlivia. Dept. Beni. Prov. Marbán, near road from Camiaco to San Lorenzo, $15^{\circ} 14^{\prime} 49^{\prime \prime} \mathrm{S}, 64^{\circ} 49^{\prime} 11^{\prime \prime} \mathrm{W}, 160 \mathrm{~m}$, lowland Amazon forest surrounded by los Llanos de Moxos savanna, corticolous, 30 Aug. 2012, MK 11951 (LPB, UGDA); Dept. Santa Cruz. Prov. Guarayos, RN de Vida Silvestre Ríos Blanco y Negro, Virgen de Pilar

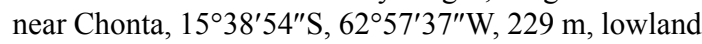
Amazon forest, corticolous, 22 July 2009, AF \& PR 13143 (KRAM, LPB).

Diorygma intermedium Kalb, Staiger \& Elix

Kukwa et al. (2013) reported this species from four localities in Bolivia.

Specimens examined. BOliVia. Dept. Santa Cruz. Prov. Guarayos, RN de Vida Silvestre Ríos Blanco y Negro, Plan de Manejo AISU, $15^{\circ} 01^{\prime} 49^{\prime \prime} \mathrm{S}$, $62^{\circ} 46^{\prime} 36^{\prime \prime} \mathrm{W}, 236 \mathrm{~m}$, lowland Amazon forest, corticolous, 25 July 2009, AF 14200 \& PR (KRAM, LPB).

\section{Diorygma poitaei (Fée) Kalb, Staiger \& Elix}

This Neotropical lichen was recently reported from two localities in Bolivia (Kukwa et al. 2013).

Specimens examined. BOliVia. Dept. Beni. Prov. Cercado, Casa del Tigre near Chuchini, $14^{\circ} 43^{\prime} 17^{\prime \prime} \mathrm{S}$, $64^{\circ} 56^{\prime} 53^{\prime \prime} \mathrm{W}, 160 \mathrm{~m}$, lowland Amazon forest, corticolous, 31 Aug. 2012, MK 11997 (LPB, UGDA).

\section{"Diorygma reniforme (Fée) Kalb, Staiger \& Elix}

This species has been reported from the Neotropics in Brazil, Colombia, Cuba, British Guiana, Peru and Venezuela, and elsewhere from Cameroon and Tanzania (Kalb et al. 2004).

Specimen examined. BOLIVIA. Dept. Santa CruZ. Prov. Guarayos, RN de Vida Silvestre Ríos Blanco y

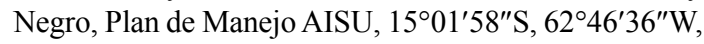
$242 \mathrm{~m}$, lowland Amazon forest, corticolous, 24 July 2009, AF 13899 \& $P R$ (LPB).

\section{"Diorygma sipmanii Kalb, Staiger \& Elix}

This Neotropical species was known previ- 
ously from Brazil, Costa Rica, Guatemala and El Salvador (Kalb et al. 2004, Cáceres 2007).

Specimen examined. BOLIVIA. Dept. Santa Cruz. Prov. Guarayos, RN de Vida Silvestre Ríos Blanco y Negro, Virgen de Pilar near Chonta, $15^{\circ} 38^{\prime} 54^{\prime \prime} \mathrm{S}$, $62^{\circ} 57^{\prime} 37^{\prime \prime} \mathrm{W}, 229 \mathrm{~m}$, lowland Amazon forest, corticolous, 22 July 2009, AF $13273 \&$ \&R (LPB).

\section{Diploschistes actinostomus (Ach.) Zahlbr.}

This species was reported in Bolivia from only one locality (Feuerer \& Sipman 2005)

Specimens examined. BOliVia. Dept. La Paz. Prov. Camacho, Pacoamba cerca Wila Kala, $15^{\circ} 24^{\prime} 40^{\prime \prime} \mathrm{S}$, $69^{\circ} 04^{\prime} 24^{\prime \prime} \mathrm{W}, 4283 \mathrm{~m}$, Puna Húmeda, on sandstone, 6 July 2010, AF 17740 \& PR (KRAM); Prov. Manco Kapac, near Copacabana Mt. Horca del Inca, $16^{\circ} 10^{\prime} 15^{\prime \prime} \mathrm{S}$, $69^{\circ} 05^{\prime} 05^{\prime \prime} \mathrm{W}, 3974 \mathrm{~m}$, high Andean Puna, on volcanic rock, 18 June 2006, $A F 8637$ (LPB).

\section{${ }^{* *}$ Diploschistes caesioplumbeus (Nyl.) Vain.}

This species was known previously from Africa (e.g., Algeria, Canary Islands, Madeira, Morocco, St. Helena and Ascension Island), Asia (e.g., India, Israel, Turkey), Europe (the Azores, France, Greece, Great Britain, Ireland, Italy, Spain) and western North America (Breuss 1988; Lumbsch 1989, 2002; Follmann 1990; Pant \& Upreti 1993; Hafellner 1995; Llimona \& Hladun 2001; Yazici \& Aslan 2003; Aptroot 2008; Seaward 2010; Roux 2012; Esslinger 2014).

Specimens examined. BOliVia. Dept. La Paz. Prov. Bautista Saavedra, APOLOBAMBA, near Villa Amarca, $15^{\circ} 16^{\prime} 47^{\prime \prime} \mathrm{S}, 6^{\circ} 01^{\prime} 47^{\prime \prime} \mathrm{W}, 4643 \mathrm{~m}$, Puna Húmeda, on sandstone, 3 July 2010, $A F$ 17349, 17355 \& PR (KRAM, LPB); Prov. Camacho, Pacoamba cerca Wila Kala, $15^{\circ} 24^{\prime} 40^{\prime \prime} \mathrm{S}, 6^{\circ} 04^{\prime} 24^{\prime \prime} \mathrm{W}, 4283 \mathrm{~m}$, Puna Húmeda, on sandstone, 6 July 2010, $A F$ 17708, 17727, 17757, 17769, $17788 \&$ PR (KRAM, LPB, herb. Flakus); Prov. Franz Tamayo, APOLOBAMBA, near Puyo Puyo, $14^{\circ} 56^{\prime} 55^{\prime \prime} \mathrm{S}, 69^{\circ} 07^{\prime} 58^{\prime \prime} \mathrm{W}, 4888 \mathrm{~m}$, high Andean open vegetation, on siliceous schist, 5 July 2010, AF 17564 \& PR (KRAM, LPB); Prov. Murillo, below Potosí, on road between La Paz and Valle del Zongo, $16^{\circ} 17^{\prime} 43^{\prime \prime} \mathrm{S}, 68^{\circ} 07^{\prime} 42^{\prime \prime} \mathrm{W}, 4716 \mathrm{~m}$, high Andean vegetation, on granite rock, 28 May 2011, $A F$ 21840, 21860 \& OP (KRAM, LPB); near Cumbre pass, $16^{\circ} 19^{\prime} 06^{\prime \prime} \mathrm{S}$, $68^{\circ} 02^{\prime} 09^{\prime \prime} \mathrm{W}, 4405 \mathrm{~m}$, high Andean vegetation, on sili- ceous schist, 31 May 2011, AF \& OP 22123 (KRAM, LPB); near Cumbre pass, $16^{\circ} 19^{\prime} 18^{\prime \prime} \mathrm{S}, 68^{\circ} 04^{\prime} 42^{\prime \prime} \mathrm{W}$, $4550 \mathrm{~m}$, high Andean Puna, on siliceous rock, 17 June 2006, $A F 8530$ (herb. Flakus).

Diploschistes cinereocaesius (Sw. ex Ach.) Vain.

This species was reported from Bolivia by Feuerer et al. (1998) and Flakus et al. (2012). It appears to be a common lichen at high elevations in the Andes.

Specimens examined. BOliVia. Dept. CochaBAMBA. Prov. Quillacollo, area of Incarraya-Sipesipe, $17^{\circ} 29^{\prime} 25^{\prime \prime} \mathrm{S}, 6^{\circ} 22^{\prime} 09^{\prime \prime} \mathrm{W}, 3146 \mathrm{~m}$, semi-desert open area, on soil, 17 Dec. 2004, AF 4977 (LPB); DEPT. LA Paz. Prov. Camacho, cerca de la comunidad de Ulla Ulla, en el cerro Wilamuku, $15^{\circ} 02^{\prime} 04^{\prime \prime} \mathrm{S}, 69^{\circ} 11^{\prime} 53^{\prime \prime} \mathrm{W}$, $4739 \mathrm{~m}$, vegetación altoandina, terrestre, $4 \mathrm{Dec}$. 2007, PR 272 (B, LPB); near Villa Cala, 15²5'53"S, $69^{\circ} 05^{\prime} 07^{\prime \prime} \mathrm{W}, 4250 \mathrm{~m}$, high Andean vegetation, on soil, 19 May 2011, AF 21236 \& OP (KRAM, LPB); Prov. Franz Tamayo, APOLOBAMBA, Socondori Chico near Ulla Ulla, $15^{\circ} 00^{\prime} 38^{\prime \prime} \mathrm{S}, 69^{\circ} 13^{\prime} 48^{\prime \prime} \mathrm{W}, 4479 \mathrm{~m}$, high Andean open vegetation, on soil, 4 July 2010, $A F 17473$ \& $P R$ (KRAM, LPB); Prov. Murillo, near Cumbre pass, $16^{\circ} 19^{\prime} 18^{\prime \prime} \mathrm{S}, 68^{\circ} 04^{\prime} 42^{\prime \prime} \mathrm{W}, 4550 \mathrm{~m}$, high Andean Puna, on soil and terricolous bryophytes, 17 June 2006, $A F 8533$ (KRAM, LPB, herb. Flakus); Valle del Zongo, Laguna Viscachani, $16^{\circ} 11^{\prime} 54^{\prime \prime} \mathrm{S}, 68^{\circ} 07^{\prime} 33^{\prime \prime} \mathrm{W}, 3862 \mathrm{~m}$, Páramo Yungueño, on soil, 30 May 2011, AF 22085, 22103 \& $O P$ (KRAM, LPB); Prov. Sud Yungas, Mururata, bajo la Mina Bolsa Negra, $16^{\circ} 35^{\prime} \mathrm{S}, 67^{\circ} 45^{\prime} \mathrm{W}, 4000 \mathrm{~m}$, saxicolous, 1995, J. Gonzáles 106 (B, LPB); ibidem, pasando por el pueblo de Tres Rios, $16^{\circ} 35^{\prime} \mathrm{S}, 67^{\circ} 45^{\prime} \mathrm{W}$, $3851 \mathrm{~m}$, saxicolous, 6 Apr. 1995, J. Gonzáles 380 (B, LPB); Dept. Santa Cruz. Prov. Manuel María Caballero, Siberia, $17^{\circ} 49^{\prime} 38^{\prime \prime} \mathrm{S}, 64^{\circ} 45^{\prime} 14^{\prime \prime} \mathrm{W}, 3000 \mathrm{~m}$, open area near Yungas cloud forest, on soil, 11 Dec. 2004, $A F$ $4474 \& 4478$ (LPB, herb. Flakus); Dept. TariJa. Prov.

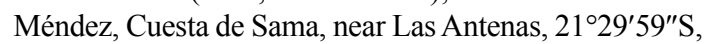
$64^{\circ} 54^{\prime} 46^{\prime \prime} \mathrm{W}, 3800 \mathrm{~m}$, high Andean vegetation, 6 Aug. 2012, MK 10958 (LPB, UGDA).

\section{*Diploschistes diacapsis (Ach.) Lumbsch}

This is a widely distributed species known from Africa (e.g., Algeria, Canary Islands, Morocco, Tunisia), Asia (e.g., China, Cyprus, India, Iran, Mongolia, Saudi Arabia, Syria), Australia, Europe (e.g., Albania, Azores, Bosnia and Herzegovina, France, Germany, Greece, Spain), North America (Mexico, 
USA) and South America (Chile, Colombia, Peru) (Schubert \& Klement 1971; Lumbsch 1989; Pant \& Upreti 1993; Hafellner 1995, 2007; Galloway \& Quilhot 1998; Litterski \& Mayrhofer 1998; Llimona \& Hladun 2001; Scholz 2001; John et al. 2004; Seaward et al. 2008; Sipman et al. 2008; Bilovitz \& Mayrhofer 2011; Roux 2012; McCarthy 2013; Esslinger 2014).

Specimens examined. BOliVia. Dept. La Paz. Prov. Omasuyos, El Dragon hill near Chahualla, $15^{\circ} 51^{\prime} 17^{\prime \prime} \mathrm{S}, 69^{\circ} 00^{\prime} 40^{\prime \prime} \mathrm{W}, 3850 \mathrm{~m}$, Puna Húmeda, on siliceous rock, 6 July 2010, AF $17836 \&$ \&R (KRAM, LPB, herb. Flakus).

"Diploschistes diploschistoides (Vain.) G. Salisb.

This species was reported previously from South Africa, Zimbabwe, Australia, and in South America from Brazil and Colombia (Guderley \& Lumbsch 1996; McCarthy 2013).

Specimens examined. BOliVia. Dept. Tarija. Prov. Aniceto Arce, Papachacra, near Papachacra valley, $21^{\circ} 41^{\prime} 14^{\prime \prime} \mathrm{S}, 64^{\circ} 30^{\prime} 19^{\prime \prime} \mathrm{W}, 2050 \mathrm{~m}$, open vegetation with shrubs, on sandstone, 7 Aug. 2012, AF 23911 (LPB, herb. Flakus).

\section{*Diploschistes euganeus (A. Massal.) Zahlbr.}

Reported in South America only from Brazil and Chile, and elsewhere from Africa (Algeria, Cape Verde, Republic of South Africa, St. Helena), Asia (Taiwan), Australia (mainland Australia, New Zealand) and Europe (France, Greece, Italy, Poland, Spain) (Lumbsch 1989; Mies \& Lumbsch 1990; Guderley \& Lumbsch 1996; Galloway \& Quilhot 1998; Llimona \& Hladun 2001; Fałtynowicz 2003; Aptroot 2008; Roux 2012; McCarthy 2013).

Specimens examined. BOLIVIA. Dept. CochaBAMBA. Prov. Quillacollo, area of Incarraya-Sipesipe, $17^{\circ} 28^{\prime} 39^{\prime \prime} \mathrm{S}, 66^{\circ} 21^{\prime} 43^{\prime \prime} \mathrm{W}, 2846 \mathrm{~m}$, semi-desert open area, on sandstone, 17 Dec. 2004, AF 4922 (LPB, herb. Flakus).

\section{"Diploschistes hypoleucus Zahlbr.}

This rarely reported species is known from Brazil, Peru and Venezuela in South America, and elsewhere in Oceania (Hawaii), continental North America, the Caribbean (Puerto Rico) and the Republic of South Africa (Lumbsch 1989; Guderley \& Lumbsch 1996; Marcano et al. 1996; Elix \& McCarthy 1998; Esslinger 2014).

Specimens examined. BOLIVIA. Dept. Cochabamba. Prov. Carrasco, CARRASCO, La Cumbre, El Camino de las Nubes, $17^{\circ} 17^{\prime} 46^{\prime \prime} \mathrm{S}, 65^{\circ} 43^{\prime} 56^{\prime \prime} \mathrm{W}, 4100 \mathrm{~m}$, Páramo Yungueño, on soil, 19 Aug. 2012, MK 11806 (LPB, UGDA); Prov. Quillacollo, area of IncarrayaSipesipe, $17^{\circ} 29^{\prime} 25^{\prime \prime} \mathrm{S}, 66^{\circ} 22^{\prime} 09^{\prime \prime} \mathrm{W}, 3146 \mathrm{~m}$, semi-desert open area, on soil, 17 Dec. 2004, AF 4964 (KRAM, LPB, herb. Flakus); DePt. LA Paz. Prov. Larecaja, la Cumbre de Sorata, 15 53'06"S, 68 38 $55^{\prime \prime} \mathrm{W}, 4210 \mathrm{~m}$, high Andean open vegetation, on soil, 13 May 2011, $A F 20256$ \& $O P$ (KRAM, LPB).

"Diploschistes muscorum (Scop.) R. Sant. subsp. bartletii Lumbsch

This taxon is less common than D. muscorum subsp. muscorum and has a remarkable Gondwanaland distribution. It was known previously from Australia, India, Lesotho, the Republic of South Africa, and in South America from Argentina, Brazil, Chile, Colombia and Ecuador (Galapagos Islands) (Pant \& Upreti 1993; Guderley \& Lumbsch 1996; Elix \& McCarthy 1998; Galloway \& Quilhot 1998; Aptroot 2002; Calvelo \& Liberatore 2002; Sipman et al. 2008; McCarthy 2013).

Specimens examined. BOliVia. Dept. La Paz. Prov. Omasuyos, El Dragon hill near Chahualla, $15^{\circ} 51^{\prime} 17^{\prime \prime} \mathrm{S}, 69^{\circ} 00^{\prime} 40^{\prime \prime} \mathrm{W}, 3850 \mathrm{~m}$, Puna Húmeda, on soil, 6 July 2010, AF 17837.1 \& PR (KRAM, LPB); DePt. LA PAZ. Prov. Nor Yungas, near Pongo, near the Coroico-La Paz road, 16 $19^{\prime} 28^{\prime \prime} \mathrm{S}, 67^{\circ} 57^{\prime} 21^{\prime \prime} \mathrm{W}, 3822 \mathrm{~m}$, Páramo Yungueño, on soil, 25 May 2011, AF 21736 $\& O P(\mathrm{LPB})$.

"Diploschistes muscorum (Scop.) R. Sant. subsp. muscorum

This widely distributed taxon is known from Africa (Cape Verde), Asia (India, Iran), Europe (e.g., Bosnia and Herzegovina, Finland, France, Norway, Poland, Sweden), North America and South America (Argentina, Chile, Colombia, Ecuador) (Mies \& Lumbsch 1990; Pant \& Upreti 1993; Elix \& McCarthy 1998; Calvelo \& Lib- 
eratore 2002; Fałtynowicz 2003; Santesson et al. 2004; Nöske et al. 2007; Seaward et al. 2008; Sipman et al. 2008; Bilovitz \& Mayrhofer 2011; Roux 2012; Esslinger 2014).

Specimens examined. BOliVia. Dept. La Paz. Prov. Bautista Saavedra, km 162 on road from Apolo to Charazani, la Cruz Charazani-Pelechuco, $15^{\circ} 15^{\prime} 00^{\prime \prime} \mathrm{S}$, $69^{\circ} 02^{\prime} 51^{\prime \prime} \mathrm{W}, 4545 \mathrm{~m}$, high Andean vegetation, on soil, 19 May 2011, $A F 21160$ \& OP (LPB); DePt. Oruro. SAJAMA, Ladera de la Quebrada Kohniri. $18^{\circ} 07^{\prime} 07^{\prime \prime}$, $68^{\circ} 56^{\prime} 06^{\prime \prime} \mathrm{W}, 4309 \mathrm{~m}$, Polylepis tarapacana forest, on rock, 21 Nov. 2007, RIM et al. 4380, 4385 (B, LPB); ibidem, $18^{\circ} 07^{\prime} 08^{\prime \prime} \mathrm{S}, 68^{\circ} 56^{\prime} 05^{\prime \prime} \mathrm{W}, 4406 \mathrm{~m}$, on rock, 21 Nov. 2007, RIM et al. 4391 (B, LPB); Prov. Sajama, SAJAMA, Jecha K'ala $18^{\circ} 09^{\prime} 52^{\prime \prime} \mathrm{S}, 68^{\circ} 49^{\prime} 08^{\prime \prime} \mathrm{W}, 4184 \mathrm{~m}$, Puna Sureña, on soil, 20 June 2010, AF 16679.1, 16684 $\& P R$ (KRAM, LPB).

\section{Dyplolabia afzeli (Ach.) A. Massal.}

This easily recognizable species is perhaps the commonest member of the Graphidaceae in Bolivia. It was previously reported from there by Kalb \& Staiger (2000), Staiger (2002), Flakus \& Wilk (2006), Flakus (2008) and Flakus et al. (2012).

Specimens examined. BOliVia. Dept. La Paz. Prov. Iturralde, near San Pedro, $13^{\circ} 43^{\prime} 59^{\prime \prime} \mathrm{S}, 68^{\circ} 00^{\prime} 38^{\prime \prime} \mathrm{W}$, $250 \mathrm{~m}$, savannah, corticolous, 28 July 2008, MK 6897 (LPB, UGDA); Tumupasa, $14^{\circ} 08^{\prime} 51^{\prime \prime} \mathrm{S}, 67^{\circ} 53^{\prime} 34^{\prime \prime} \mathrm{W}$, $350 \mathrm{~m}$, garden, on mango tree, 31 Aug. 2008, MK 6996 (LPB, UGDA).

\section{*Fissurina triticea (Nyl.) Staiger}

This species was previously reported from Australia, Madeira, New Zealand, Reunion, the Azores, and in the Neotropics from Colombia, Costa Rica, Dominica, Jamaica and Mexico (Wirth \& Hale 1978; Hafellner 1995; Staiger 2002; Sipman 2006; Sipman et al. 2008).

Specimens examined. BOliVia. Dept. La Paz. Prov. Nor Yungas, near Nogalani, $16^{\circ} 12^{\prime} 57^{\prime \prime} \mathrm{S}$, $67^{\circ} 49^{\prime} 15^{\prime \prime} \mathrm{W}, 2168 \mathrm{~m}$, Yungas secondary cloud forest, corticolous, 23 May 2011, US (LPB, herb. Schiefelbein 3069, 3070); DePt. Santa CruZ. Prov. Manuel María Caballero, El Camino de Orquideas, $17^{\circ} 49^{\prime} 20^{\prime \prime} \mathrm{S}$, $64^{\circ} 42^{\prime} 31^{\prime \prime} \mathrm{W}, 2340 \mathrm{~m}$, Yungas cloud forest, corticolous, 17 Aug. 2012, MK 11564 (LPB, UGDA).

\section{*Graphis caesiella Vain.}

This is a pantropical species reported in the Neotropics from Brazil, Peru and the USA (Louisiana, Florida) (Staiger 2002; Cáceres 2007; Lücking et al. 2009; Seavey \& Seavey 2011; Rivas Plata \& Lücking 2013), and also from the Philippines (LinsanganTabaquero et al. 2013) and Australia (Archer 2009).

Specimen examined. BOliVia. Dept. Santa Cruz. Prov. Ichilo, AMBORÓ, Macuñucu, $17^{\circ} 43^{\prime} 38^{\prime \prime} \mathrm{S}$, $63^{\circ} 35^{\prime} 37^{\prime \prime} \mathrm{W}, 460 \mathrm{~m}$, Amazon forest, on twigs, 6 June 2011, MK $9696 a$ (LPB).

\section{${ }^{*}$ Graphis cincta (Pers.) Aptroot}

This species was previously reported in the Neotropics from Costa Rica, the Dominican Republic, Paraguay, the USA (Florida), and elsewhere from the Philippines and Taiwan (Lücking et al. 2008, 2009; Seavey \& Seavey 2011) and from Australia and Japan (Archer 2009).

Specimens examined. BOliVia. Dept. La Paz. Prov. Sud Yungas, Alto Beni, San Antonio. 15 $38^{\prime} 13^{\prime \prime}$, $67^{\circ} 12^{\prime} 09^{\prime \prime} \mathrm{W}, 405 \mathrm{~m}$, on Theobroma cacao, 29 June 1999, N. Derakshani 12 (B, GOET).

${ }^{* *}$ Graphis daintreensis (A. W. Archer) A. W. Archer

Previously known only from Australia and the Solomon Islands (Archer 2009).

Specimens examined. BOliVia. Dept. Beni. Prov. Vaca Díez, Cachuela Esperanza, $10^{\circ} 32^{\prime} 09^{\prime \prime} \mathrm{S}$, $65^{\circ} 34^{\prime} 55^{\prime \prime} \mathrm{W}, 128 \mathrm{~m}$, lowland Amazon forest, corticolous, 4 June 2006, AF 7525 (KRAM, LPB).

\section{"Graphis dracaenae Vain.}

Previously known from Africa, Australia and Brazil (Archer 2009; Lücking et al. 2009).

Specimen examined. BOliVia. Dept. La Paz. Prov. Nor Yungas, COTAPATA, Santa Catalina, above Tunkini, $16^{\circ} 11^{\prime} 12^{\prime \prime} \mathrm{S}, 67^{\circ} 52^{\prime} 07^{\prime \prime} \mathrm{W}$, Yungas montane forest, corticolous, 22 May 2011, US (LPB).

\section{Graphis duplicata Ach.}

Reported only recently from three localities in Bolivia by Flakus et al. (2013) and Kukwa et al. (2103). 
Specimen examined. BOliVia. Dept. Santa Cruz. Prov. Manuel María Caballero, Siberia region near La

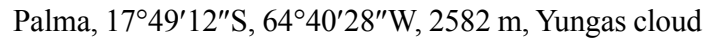
forest, corticolous, 13 Dec. 2004, AF 4642 (KRAM, LPB).

\section{**Graphis duplicatoinspersa Lücking}

The species has been reported from Costa Rica and Australia (Lücking et al. 2008, 2009).

Specimen examined. BOlivia. Dept. La Paz. Prov. Nor Yungas, COTAPATA, near Urpuma colony, $16^{\circ} 13^{\prime} 20^{\prime \prime} \mathrm{S}, 67^{\circ} 52^{\prime} 34^{\prime \prime} \mathrm{W}, 1989 \mathrm{~m}$, Yungas montane forest, corticolous, 30 June 2010, $A F 17118$ \& PR (KRAM, LPB, herb. Flakus).

\section{**Graphis emersa Müll. Arg.}

Previously reported from Australia, Costa Rica and Japan (Lücking et al. 2008, 2009).

Specimen examined. BOLIVIA. Dept. La Paz. Prov. Nor Yungas, between Coroico and La Paz, 16 ${ }^{\circ} 13^{\prime} 33^{\prime \prime} \mathrm{S}$, $67^{\circ} 49^{\prime} 20^{\prime \prime} \mathrm{W}, 2600 \mathrm{~m}$, Yungas secondary cloud forest, corticolous, 23 May 2011, US (LBP).

\section{Graphis aff. evirescens (Redinger) Lücking}

To our knowledge this species was previously reported only from the type locality in Brazil (Lücking et al. 2009).

With Lücking et al. (2009), our material keys out as $G$. evirescens, characterized by a green thallus, stellately branched lirellae with an apically thin, complete thalline margin, 8-spored asci and small muriform ascospores, 12-18 $\times$ 7-14 $\mu \mathrm{m}$ (Redinger 1935; Lücking et al. 2009). In our specimen the lirellae are unbranched, the asci 2-spored and the ascospores larger (30-44 $\times$ $13-17 \mu \mathrm{m})$. It is not clear to us if this should be considered an instance of infraspecific variation, such as known in other Graphis species (Lücking et al. 2009), so the present report should be considered tentative.

Specimen examined. BOliVia. Dept. La Paz. Prov. Nor Yungas, COTAPATA, between Tunkini and Chairo villages, above Tunkini, $16^{\circ} 11^{\prime} \mathrm{S}, 67^{\circ} 52^{\prime} \mathrm{W}$, $2400 \mathrm{~m}$, Yungas montane forest, corticolous, 22 May 2011, MK 9101 (LPB, UGDA).
*Graphis handelii Zahlbr.

Previously reported from Brazil, China, Costa Rica, India and Paraguay (Lücking et al. 2008, 2009).

Specimens examined. BOliVia. Dept. La Paz. Prov. Nor Yungas, between Coroico and $\mathrm{La} \mathrm{Paz}$, $16^{\circ} 13^{\prime} 33^{\prime \prime} \mathrm{S}, 67^{\circ} 49^{\prime} 20^{\prime \prime} \mathrm{W}, 2600 \mathrm{~m}$, Yungas secondary cloud forest, corticolous, 23 May 2011, US (LBP); Dept. Santa Cruz. Prov. Guarayos, RN de Vida Silvestre Ríos Blanco y Negro, Plan de Manejo AISU, $15^{\circ} 01^{\prime} 49^{\prime \prime} \mathrm{S}, 62^{\circ} 46^{\prime} 36^{\prime \prime} \mathrm{W}, 236 \mathrm{~m}$, lowland Amazon forest, corticolous, 25 July 2009, $A F 14254 \& P R$ (LPB).

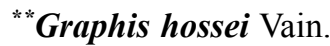

Previously this species was known only from Asia (China, Indonesia, Philippines and Thailand) (Lücking et al. 2009).

Specimen examined. BOliVia. Dept. La Paz. Prov. Nor Yungas, COTAPATA, Chairo, $16^{\circ} 12^{\prime} 07^{\prime \prime} \mathrm{S}$, $67^{\circ} 50^{\prime} 05^{\prime \prime} \mathrm{W}, 1300 \mathrm{~m}$, Yungas lower montane forest, corticolous, 21 May 2011, US (LPB).

\section{Graphis illinata Eschw.}

Reported from Bolivia by Flakus \& Wilk (2006).

Specimens examined. BOliVia. Dept. La Paz. Prov. Nor Yungas, near Nogalani, between Coroico and La Paz, 16 $6^{\circ} 12^{\prime} 57^{\prime \prime} \mathrm{S}, 67^{\circ} 49^{\prime} 15^{\prime \prime} \mathrm{W}, 2168 \mathrm{~m}$, Yungas secondary cloud forest, corticolous, 23 May 2011, US (LPB, herb. Schiefelbein 3453); Prov. Nor Yungas, COTAPATA, Santa Catalina, above Tunkini, $16^{\circ} 11^{\prime} 12^{\prime \prime} \mathrm{S}$, $67^{\circ} 52^{\prime} 07^{\prime \prime} \mathrm{W}$, Yungas montane forest, corticolous, 22 May 2011, US (LPB, herb. Schiefelbein 3057).

\section{**Graphis immersella Müll. Arg.}

Known previously from Australia, China, the Philippines, New Caledonia, the Solomon Islands and Vanuatu (Archer 2009; Lücking et al. 2009; Linsangan-Tabaquero et al. 2013).

Specimens examined. BOliVia. Dept. Santa Cruz. Prov. Guarayos, RN de Vida Silvestre Ríos Blanco y Negro, Virgen de Pilar near Chonta, $15^{\circ} 38^{\prime} 54^{\prime \prime} \mathrm{S}$, $62^{\circ} 57^{\prime} 37^{\prime \prime} \mathrm{W}, 229 \mathrm{~m}$, lowland Amazon forest, corticolous, 22 July 2009, AF $13217 \&$ PR (KRAM, LPB). 


\section{"Graphis librata Vain.}

In the Neotropics this species was found in Brazil, Costa Rica, Panama and Venezuela, and elsewhere in Australia, New Zealand, the Philippines, South Africa and the USA (Archer 2001; Staiger 2002; Lücking et al. 2008, 2009; Neuwirth 2008; Seavey \& Seavey 2011; Boom \& Sipman 2013; Linsangan-Tabaquero et al. 2013).

Specimens examined. BOliVia. Dept. Santa Cruz. Prov. Cordillera, KAA-IYA, near Peto Blanco, $18^{\circ} 56^{\prime} 26^{\prime \prime} \mathrm{S}, 60^{\circ} 22^{\prime} 39^{\prime \prime} \mathrm{W}, 340 \mathrm{~m}$, Chiquitano forest, on twig, 5 Dec. 2011, MK 10644 (LPB, UGDA).

\section{Graphis lumbricina Vain.}

Previously reported from four localities in Bolivia (Flakus et al. 2103; Kukwa et al. 2013).

Specimens examined. BOliVia. Dept. Santa Cruz. Prov. Manuel María Caballero, near Siberia, $17^{\circ} 49^{\prime} 38^{\prime \prime} \mathrm{S}, 64^{\circ} 44^{\prime} 45^{\prime \prime} \mathrm{W}, 3950 \mathrm{~m}$, open Yungas cloud forest, corticolous, 16 Aug. 2012, MK 11477 (LPB, UGDA).

\section{"Graphis paralleloides Cáceres \& Lücking}

Reported from Brazil and India (Cáceres 2007; Lücking et al. 2009).

According to Cáceres (2007) and Lücking et al. (2009) this species produces transversely septate ascospores measuring 30-40 × 7-8 $\mu \mathrm{m}$. In one collections cited below ( $M K 11565)$ the ascospores were 38-50 $\times 13-14 \mu \mathrm{m}$, and in the other $(M K$ 9792a) 25-40 × 7-12 $\mu \mathrm{m}$, but otherwise both collection had the same morphology of ascomata (striatula-morph) as presented by Cáceres (2007) and Lücking et al. (2009). Considerable variation of ascospore dimensions is known in other Graphis species as well (e.g., in Graphis conferta Zenker or G. ovata (Fée) A. Massal.; Lücking et al. 2009), so here we widen the circumscription of G. paralleloides instead of distinguishing a new species.

Specimens examined. BOliVia. Dept. Santa Cruz. Prov. Florida, AMBORÓ, above la Yunga village, senda Los Helechos, $18^{\circ} 03^{\prime} 30^{\prime \prime} \mathrm{S}, 63^{\circ} 54^{\prime} 36^{\prime \prime} \mathrm{W}$, $2330 \mathrm{~m}$, Yungas cloud forest, on dead twig, 7 June 2011, MK $9792 a$ (LPB); Prov. Manuel María Caballero, El Camino de Orquideas, $17^{\circ} 49^{\prime} 20^{\prime \prime} \mathrm{S}, 64^{\circ} 42^{\prime} 31^{\prime \prime} \mathrm{W}$,
2340 m, Yungas cloud forest, corticolous, 17 Aug. 2012, MK 11565 (LPB, UGDA).

\section{*Graphis paraserpens Lizano \& Lücking}

So far reported from Brazil and Costa Rica (Cáceres 2007; Lücking et al. 2008, 2009).

Specimens examined. BOliVia. Dept. Santa Cruz. Prov. Guarayos, RN de Vida Silvestre Ríos Blanco y Negro, Plan de Manejo AISU, $15^{\circ} 01^{\prime} 49^{\prime \prime} \mathrm{S}$, $62^{\circ} 46^{\prime} 36^{\prime \prime} \mathrm{W}, 236 \mathrm{~m}$, lowland Amazon forest, corticolous, 25 July 2009, AF $14250 \&$ PR (KRAM, LPB).

\section{"Graphis phaeospora Vain.}

So far known only from Brazil and Ecuador (Staiger 2002; Nöske et al. 2007).

Specimens examined. BOliVia. Dept. CochaBAMBA. Prov. Carrasco, CARRASCO, near Sehuencas, $17^{\circ} 30^{\prime} 12^{\prime \prime} \mathrm{S}, 65^{\circ} 16^{\prime} 30^{\prime \prime} \mathrm{W}, 2220 \mathrm{~m}$, montane cloud forest, along road, corticolous, 21 July 2008, MK 6395 (LPB, UGDA); Dept. Santa Cruz. Prov. Manuel María Caballero, Siberia region near La Palma, $17^{\circ} 49^{\prime} 12^{\prime \prime} \mathrm{S}$, $64^{\circ} 40^{\prime} 28^{\prime \prime} \mathrm{W}, 2582 \mathrm{~m}$, Yungas cloud forest, on sandstone, 13 Dec. 2004, AF 4641 (LPB).

\section{"Graphis pitmanii Rivas Plata \& Lücking}

This species was only recently described from Peru (Rivas Plata \& Lücking 2013).

Specimens examined. BOLIVIA. Dept. La Paz. Prov. Bautista Saavedra, Calzada, $15^{\circ} 09^{\prime} 06^{\prime \prime} \mathrm{S}, 68^{\circ} 30^{\prime} 24^{\prime \prime} \mathrm{W}$, $834 \mathrm{~m}$, Preandean Amazon secondary forest, corticolous, 17 May 2011, US (LPB, herb. Schiefelbein 2012); Prov.

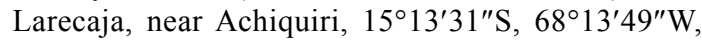
$1071 \mathrm{~m}$, Yungas secondary forest, corticolous, 16 May 2011, US (LPB, herb. Schiefelbein 2988).

\section{“Graphis puiggarii (Müll. Arg.) Lücking}

Known before from Brazil, Costa Rica and Paraguay (Lücking et al. 2008, 2009).

Specimens examined. BOlivia. Dept. La Paz. Prov. Franz Tamayo, near Correo, $14^{\circ} 59^{\prime} 06^{\prime \prime}$ S, $68^{\circ} 26^{\prime} 09^{\prime \prime} \mathrm{W}, 1632 \mathrm{~m}$, Yungas montane forest, open area, corticolous, 17 May 2011, US (LPB, herb. Schiefelbein 3002); Prov. Larecaja, near Incachaca, $15^{\circ} 17^{\prime} 48^{\prime \prime} \mathrm{S}$, $68^{\circ} 26^{\prime} 32^{\prime \prime} \mathrm{W}, 1100 \mathrm{~m}$, Yungas lower montane forest, corticolous, 15 May 2011, US (LPB, herb. Schiefelbein 2980); Prov. Nor Yungas, COTAPATA, Santa Catalina, 
above Tunkini, $16^{\circ} 11^{\prime} 12^{\prime \prime} \mathrm{S}, 67^{\circ} 52^{\prime} 07^{\prime \prime} \mathrm{W}$, Yungas montane forest, 22 May 2011, corticolous, US (LPB, herb. Schiefelbein 3029); Chairo, $16^{\circ} 12^{\prime} 07^{\prime \prime} \mathrm{S}, 67^{\circ} 50^{\prime} 05^{\prime \prime} \mathrm{W}$, Yungas lower montane forest, corticolous, $1300 \mathrm{~m}, 21$ May 2011, US (LPB).

\section{"Graphis rhizocola (Fée) Lücking \& Chaves}

This Neotropical species was reported previously from Brazil, Costa Rica, Peru, St. Vincent and Trinidad (Lücking et al. 2008, 2009).

Specimens examined. BOliVia. Dept. La Paz. Prov. Nor Yungas, COTAPATA, Santa Catalina, above Tunkini, $16^{\circ} 11^{\prime} 12^{\prime \prime} \mathrm{S}, 67^{\circ} 52^{\prime} 07^{\prime \prime} \mathrm{W}$, Yungas montane forest, 17 May 2011, US (LPB, herb. Schiefelbein 3056); by Sillu Tincara pre-Columbian route, $16^{\circ} 17^{\prime} 22^{\prime \prime} \mathrm{S}$,

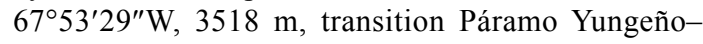
Yungas montane cloud forest, 25 May 2011, US (LPB, herb. Schiefelbein 3456).

\section{"Graphis sitiana Vain.}

A Neotropical species known previously from Brazil, Costa Rica, Panama and Peru (Lücking et al. 2008, 2009; Boom \& Sipman 2013; Rivas Plata \& Lücking 2013).

Specimens examined. BOliVia. Dept. La Paz. Prov. Sud Yungas, Alto Beni Sapecho. Cooperativa Sapecho. $15^{\circ} 32^{\prime} \mathrm{S}, 67^{\circ} 20$ 'W, $500 \mathrm{~m}$, on branches, 2 Aug. 1999, A. Acebey \& T. Krömer 997 (B, LPB).

\section{**Graphis subchrysocarpa Lücking}

This species is known from Central America (Costa Rica) and Africa (Lücking et al. 2008, 2009).

Specimens examined. BOLIVIA. Dept. Santa Cruz. Prov. Guarayos, RN de Vida Silvestre Ríos Blanco y Negro, Plan de Manejo AISU, $15^{\circ} 01^{\prime} 49^{\prime \prime} \mathrm{S}$, $62^{\circ} 46^{\prime} 36^{\prime \prime} \mathrm{W}, 236 \mathrm{~m}$, lowland Amazon forest, corticolous, 25 July 2009, AF 14272 \& PR (KRAM, LPB, herb. Flakus).

\section{*Graphis subradiata (Nyl.) Lücking}

Known only from the Neotropics and previously reported from Brazil, Colombia and Costa Rica (Lücking et al. 2008, 2009).

Specimens examined. BOliVia. Dept. La Paz.
Prov. Nor Yungas, COTAPATA, between Tunkini and Chairo, $16^{\circ} 11^{\prime} 00^{\prime \prime} \mathrm{S}, 67^{\circ} 52^{\prime} 00^{\prime \prime} \mathrm{W}$, corticolous, 22 May 2011, US (LPB).

\section{*Graphis supracola A. W. Archer}

A Pantropical species known from Australia, Cambodia, Japan and Thailand, and in the Neotropics from Brazil, Colombia and the USA (Florida) (Archer 2001, 2012; Lücking et al. 2009; Nakanishi et al. 2010; Seavey \& Seavey 2011).

Specimens examined. BOLIVIA. Dept. Beni. Prov. Marbán, near San Lorenzo, $15^{\circ} 12^{\prime} 59^{\prime \prime} \mathrm{S}, 64^{\circ} 47^{\prime} 08^{\prime \prime} \mathrm{W}$, $160 \mathrm{~m}$, lowland Amazon forest surrounded by los Llanos de Moxos savanna, on twigs, 28 Aug. 2012, MK 11897 (LPB, UGDA).

\section{"Hemithecium laubertianum (Fée) Staiger}

This rare species is known from Dominica, Peru and Australia (Staiger 2002; Archer 2009).

Specimens examined. BOliVia. Dept. Beni. Prov. Cercado, Casa del Tigre near Chuchini, $14^{\circ} 43^{\prime} 17^{\prime \prime} \mathrm{S}$, $64^{\circ} 56^{\prime} 53^{\prime \prime} \mathrm{W}, 160 \mathrm{~m}$, lowland Amazon forest, corticolous, 31 Aug. 2012, MK 11984 (LPB, UGDA, herb. Flakus).

\section{${ }^{* * *}$ Leiorreuma lyellii $(\mathrm{Sm}$.) Staiger}

Phaeographis lyellii (Sm.) Zahlbr.

Previously reported from Great Britain, France, Ireland, Spain and Macaronesia (Madeira), and in the Neotropics only from Costa Rica (Llimona \& Hladun 2001, Staiger 2002; Breuss \& Neuwirth 2007; Benfield et al. 2009; Seaward 2010; Roux 2012).

Specimens examined. BOliVia. Dept. La Paz. Prov. Nor Yungas, near Chuspipata, 16 $17^{\prime} 09^{\prime \prime} \mathrm{S}$, $67^{\circ} 51^{\prime} 00^{\prime \prime} \mathrm{W}, 3228 \mathrm{~m}$, Yungas cloud forest with Ericaceae, corticolous, 24 May 2011, US (LPB, herb. Schiefelbein 3111).

${ }^{*}$ Pallidogramme chrysenteron (Mont.) Staiger, Kalb \& Lücking

This species was reported in the Neotropics from Brazil, Colombia, Costa Rica, Guyana and Panama, and elsewhere from Australia, the Philippines and Thailand (Staiger 2002; Wolseley et al. 
2002; Sipman et al. 2008; Boom \& Sipman 2013; Linsangan-Tabaquero et al. 2013).

Specimens examined. BOLIVIA. Dept. La Paz. Prov. Bautista Saavedra, Calzada, $15^{\circ} 09^{\prime} 06^{\prime \prime} \mathrm{S}, 68^{\circ} 30^{\prime} 24^{\prime \prime} \mathrm{W}$, $835 \mathrm{~m}$, Preandean Amazon secondary forest, corticolous, 17 May 2011, MK 8806 (LPB, UGDA).

\section{Phaeographis leprieurii (Mont.) Staiger}

Previously known from only a single locality in Bolivia (Kukwa et al. 2013).

Specimens examined. BOliVia. Dept. La Paz. Prov. Abel Iturralde, Tumupasa village, $14^{\circ} 08^{\prime} 51^{\prime \prime} \mathrm{S}$, $67^{\circ} 53^{\prime} 34^{\prime \prime} \mathrm{W}, 350 \mathrm{~m}$, garden in the village, on mango tree, 31 Aug. 2008, MK 6998 (LPB, UGDA).

\section{Phaeographis scalpturata (Ach.) Staiger}

This species was reported from Bolivia before by Nylander (1859) and Flakus et al. (2013).

Specimens examined. BOliVia. Dept. Beni. Prov. Ballivian, Palmar village, $14^{\circ} 58^{\prime} 56^{\prime \prime} \mathrm{S}, 67^{\circ} 05^{\prime} 16^{\prime \prime} \mathrm{W}$, $250 \mathrm{~m}$, by road, corticolous, 2 Aug. 2008, MK 7109, 7112 (LPB, UGDA); Dept. La PAZ. Prov. Nor Yungas, Coroico, $16^{\circ} 11^{\prime} 10^{\prime \prime} \mathrm{S}, 67^{\circ} 43^{\prime} 16^{\prime \prime} \mathrm{W}, 1550 \mathrm{~m}$, Yungas montane forest, corticolous, 6 June 2010, $A F 16430.2$ $\& P R$ (KRAM, LPB); near Pacallo village, $16^{\circ} 12^{\prime} 10^{\prime \prime} \mathrm{S}$, $67^{\circ} 50^{\prime} 39^{\prime \prime} \mathrm{W}, 1360 \mathrm{~m}$, montane forest, corticolous, 3 Aug. 2008, MK 7170 (LPB, UGDA); Dept. Santa Cruz. Prov. Guarayos, RN de Vida Silvestre Ríos Blanco y Negro, Plan de Manejo AISU, $15^{\circ} 01^{\prime} 49^{\prime \prime} \mathrm{S}, 6^{\circ} 46^{\prime} 36^{\prime \prime} \mathrm{W}$, 236 m, lowland Amazon forest, corticolous, 25 July 2009, $A F 14238,14242 \& P R$ (KRAM, LPB); ibidem, $15^{\circ} 01^{\prime} 58^{\prime \prime} \mathrm{S}, 62^{\circ} 46^{\prime} 36^{\prime \prime} \mathrm{W}, 242 \mathrm{~m}, 24$ July $2009, A F$ 13886 \& PR (LPB); Virgen de Pilar near Chonta, $15^{\circ} 38^{\prime} 54^{\prime \prime} \mathrm{S}, 62^{\circ} 57^{\prime} 37^{\prime \prime} \mathrm{W}, 229 \mathrm{~m}$, lowland Amazon forest, corticolous, 22 July 2009, $A F 13257 \& P R$ (LPB).

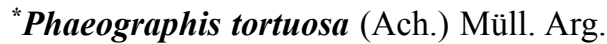

Reported so far from the Caribbean, Brazil and Panama (Staiger 2002; Cáceres 2007; Boom \& Sipman 2013).

Our material agrees anatomically and chemically with the description given by Staiger (2002) but the thallus is rather smooth (as also pictured by Cáceres 2007) than granular. Taking under consideration the variability of thallus morphology in other Phaeographis species [e.g., P. intricans
(Leight.) Kalb and P. scalpturata; pictures in, e.g., Lücking et al. 2011; Aptroot \& Sparrius 2014), we refer our sample to $P$. tortuosa.

Specimens examined. BOliVia. Dept. La Paz. near Pacallo village, $16^{\circ} 12^{\prime} 10^{\prime \prime} \mathrm{S}, 67^{\circ} 50^{\prime} 39^{\prime \prime} \mathrm{W}, 1360 \mathrm{~m}$, montane forest, corticolous, 3 Aug. 2008, MK 7115 (LPB, UGDA).

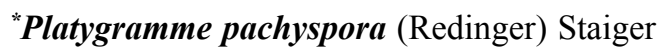

So far known only from Brazil (Staiger 2002).

Specimens examined. BOliVia. Dept. La Paz. Prov. Larecaja, near Achiquiri, $15^{\circ} 13^{\prime} 31^{\prime \prime} \mathrm{S}, 68^{\circ} 13^{\prime} 49^{\prime \prime} \mathrm{W}$, $1071 \mathrm{~m}$, Yungas secondary forest, 16 May 2011, US (LPB, herb. Schiefelbein 2988).

\section{"Platythecium serpentinellum (Nyl.) Staiger}

To our knowledge this species was reported only from Colombia (Staiger 2002; Sipman et al. 2008).

Specimens examined. BOlivia. Dept. La Paz. Prov. Nor Yungas, COTAPATA, Chairo, $16^{\circ} 12^{\prime} 07^{\prime \prime} \mathrm{S}$, $67^{\circ} 50^{\prime} 05^{\prime \prime} \mathrm{W}$, Yungas lower montane forest, $1300 \mathrm{~m}$, corticolous, 21 May 2011, US (LPB).

\section{"Reimnitzia santensis (Tuck.) Kalb}

A Pantropical species known in the Neotropics from Brazil, Costa Rica and Paraguay, and elsewhere from Australia, Mozambique and the USA (South Carolina) (Frisch 2006; Sipman et al. 2012; Esslinger 2014).

Specimens examined. BOliviA. Dept. Beni. Prov. Cercado, Casa del Tigre near Chuchini, 14² $43^{\prime} 17^{\prime \prime} \mathrm{S}$, $64^{\circ} 56^{\prime} 53^{\prime \prime} \mathrm{W}, 160 \mathrm{~m}$, lowland Amazon forest, corticolous, 31 Aug. 2012, MK 11991 (LPB, UGDA).

ACKNOWLEDGEMENTS. We are greatly indebted to Rosa I. Meneses Q., Director of Herbario Nacional de Bolivia, Universidad Mayor de San Andrés, La Paz for her kind hospitality and assistance, to a number of people who were so helpful during the field work, in particular Pamela Rodriguez Flakus (Frankfurt), and to the anonymous reviewers for helpful suggestions on the manuscript. This research received support from the Polish National Centre for Research and Development (NCBiR) under the LIDER Programme for 2010-2013 (No. 92/L-1/09). 


\section{REFERENCES}

Aptroot A. 2002. New and interesting lichens and lichenicolous fungi in Brazil. Fung. Diversity 9: 15-45.

Aртrоoт A. 2008. Lichens of St Helena and Ascension Island. Bot. J. Linn. Soc. 158: 147-171.

Aptroot A. \& Sparrius L. 2014. Pictures of tropical lichens. [3 March 2014]. http://www.tropicallichens.net.

Aptroot A., Thor G., Lücking R., Elix J. A. \& Chaves J. L. 2009. The lichen genus Herpothallon reinstated. Biblioth. Lichenol. 99: 19-66.

ArCher A. W. 2001. The lichen genus Graphis (Graphidaceae) in Australia. Austral. Syst. Bot. 14: 245-271.

Archer A. W. 2009. Graphidaceae. In: P. M. McCArthy \& B. Kuchlmayr (eds), Flora of Australia. 57. Lichens 5: 84-194. ABRS and CSIRO Publishing, Collingwood.

Archer A. W. 2012. Graphis supracola A.W. Archer is not endemic to Australia. Australas. Lichenol. 71: 18-19.

Benfield B., Coppins B. J. \& James P. W. 2009. Phaeographis Müll. Arg. (1882). In: C. W. Smith, A. Артroot, B. J. Coppins, A. Fletcher, O. L. Gilbert, P. W. James \& P. A. Wolseley (eds), The lichens of Great Britain and Ireland, pp. 691-693. British Lichen Society, London.

Bilovitz P. O. \& MAYrhofer H. 2011. Catalogue of the lichenized and lichenicolous fungi of Bosnia and Herzegovina. Phyton (Horn) 51(1): 1-67.

Boom P. P. G. van den \& Sipman H. J. M. 2013. Sixty-two species of lirelliform Graphidaceae (Ascomycota) new to Panama, including four species new to science. Herzogia 26: 9-20.

Breuss O. 1988. Neue und bemerkenswerte Flechtenfunde aus Tenerife (Kanarische Inseln). Linzer Biol. Beitr. 20(2): 829-845.

Breuss O. \& Neuwirth G. 2007. Flechtenfunde im Bosque Esquinas, Costa Rica. Linzer Biol. Beitr. 39(1): 557-569.

CÁCERes M. E. S. 2007. Corticolous crustose and microfoliose lichens of northeastern Brazil. IHW-Verlag und Verlagsbuchhandlung, München.

Calvelo S. \& Liberatore S. 2002. Catálogo de los líquenes de la Argentina. Kurtziana 29(2): 7-170.

Elix J. A. \& McCARThy P. M. 1998. Catalogue of the lichens of the smaller Pacific islands. Biblioth. Lichenol. 70: 1-361.

ESSLINGER T. L. 2014. A cumulative checklist for the lichenforming, lichenicolous and allied fungi of the continental United States and Canada. North Dakota State University, Fargo. Vers. 19. [23 March 2014]. http://www.ndsu.edu/ pubweb/ esslinge/chcklst/chcklst7.htm.

Faetynowicz W. 2003. The lichens, lichenicolous and allied fungi of Poland - an annotated checklist. W. Szafer Institute of Botany, Polish Academy of Sciences, Kraków.
Feuerer T. \& Sipman H. J. M. 2005. Additions to the lichenized and lichenicolous fungi of Bolivia. Herzogia 18: 139-144.

Feuerer T., Ahti T. \& Vitikainen O. 1998. Lichenological investigations in Bolivia. In: M. P. MARCELLI \& M. R. D. SEAWARD (eds), Lichenology in Latin America: history, current knowledge and applications, pp. 71-86. CETESB, Săo Paulo.

Flakus A. 2008. Contributions to the knowledge of the lichen biota of Bolivia. 2. Polish Bot. J. 53: 145-153.

Flakus A. \& WilK K. 2006. Contribution to the knowledge of the lichen biota of Bolivia. J. Hattori Bot. Lab. 99: 307-318.

Flakus A., Etayo J., Schiefelbein U., Ahti T., JablońsKa A., Oset M., Bach K., Rodriguez Flakus P. \& Kukwa M. 2012. Contribution to the knowledge of the lichen biota of Bolivia. 4. Polish Bot. J. 53: 427-461.

Flakus A., Sipman H. J. M., Bach K., Rodriguez Flakus P., Knudsen K., Ahti T., Schiefelbein U., Palice Z., Jablońska A., Oset M., Meneses Q. R. I. \& KuKwa M. 2013. Contribution to the knowledge of the lichen biota of Bolivia. 5. Polish Bot. J. 58: 697-733.

Follmann G. 1990. Zur Kenntnis der Flechtenflora und Flechtenvegetation von Madeira und den umliegenden Inseln. I. Chorologisch-soziologischer Abriss. Courier Forschungsinst. Senckenberg 129: 91-102.

Frisch A. 2006 The lichen family Thelotremataceae in Africa. A revision with special consideration of the taxa from Cameroon and Tanzania. Biblioth. Lichenol. 92: 3-370.

Galloway D. J. \& Quilhot W. 1998. Checklist of Chilean lichen-forming and lichenicolous fungi. Gayana, Bot. 55(2): 111-185.

Guderley R. \& Lumbsch H. T. 1996. The lichen genus Diploschistes in South Africa (Thelotremataceae). Mycotaxon 58: 269-292.

HAFELlNER J. 1995. A new checklist of lichens and lichenicolous fungi of insular Laurimacaronesia including a lichenological bibliography for the area. Fritschiana 5: 1-132.

HAFELlNER J. 2007. Checklist and bibliography of lichenized and lichenicolous fungi so far reported from Albania (version 05-2007). Fritschiana 59: 1-18.

IBISCH P. L. \& MÉRIDA G. (eds) 2004. Biodiversity: the richness of Bolivia. State of knowledge and conservation. Ministry of Sustainable Development. Editorial FAN, Santa Cruz de la Sierra, Bolivia.

John V., Seaward M. R. D., Sipman H. J. M. \& Zedda L. 2004. Lichens and lichenicolous fungi of Syria, including a first checklist. Herzogia 17: 157-177.

Josse C., Navarro G., Comer P., Evans R., Faber-Langendoen D., Fellows M., Kittel G., Menard S., Pyne M., Reid M., Schulz K., Snow K. \& Teague J. 2003. Ecological Systems of Latin America and the Caribbean: A Working Classification of Terrestrial Systems. Nature-Serve, 
Arlington. http://www.natureserve.org/library/LACEcologicalSystems.pdf.

Kalb K. \& Staiger B. 2000. Dyplolabia Massalongo. Monographie einer vergessenen Flechtengattung. Hoppea 61: 409-422.

Kalb K., Staiger B. \& Elix J. A. 2004. A monograph of the lichen genus Diorygma - a first attempt. Symb. Bot. Upsal. 34: 133-181.

KuKwa M., Schiefelbein U. \& FlaKus A. 2013. A contribution to the lichen family Graphidaceae (Ostropales, Ascomycota) of Bolivia. Herzogia 26(2): 231-252.

Linsangan-Tabaquero A., Bawingan P. A. \& Lücking R. 2013. Key and checklist of graphidaceae lichens in the Kalahan Forest Reserve, Nueva Vizcaya, Philippines. Philipp. J. Syst. Biol. 7: 22-38.

LitTerski B. \& Mayrhofer H. 1998. Catalogue of lichenized and lichenicolous fungi of Cyprus. Stud. Geobot. 16: $57-70$.

Llimona X. \& Hladun N. L. 2001. Checklist of the lichens and lichenicolous fungi of the Iberian Peninsula and Balearic Islands. Bocconea 14: 1-581.

Lücking R., Chaves J. L., Sipman H. J. M., Umaña L. \& ApтRоот A. 2008. A first assessment of the Ticolichen biodiversity inventory in Costa Rica: The genus Graphis, with notes on the genus Hemithecium (Ascomycota: Ostropales: Graphidaceae). Fieldiana, Bot. 46: 1-131.

Lücking R., Archer A. W. \& Aptroot A. 2009. A world-wide key to the genus Graphis (Ostropales: Graphidaceae). Lichenologist 41(4): 363-452.

Lücking R., Seavey F., Common R. S., Beeching S. Q., Breuss O., Buck W. R., Crane L., Hodges M., Hodkinson B. P., Lay E., Lendemer J. C., McMullin R. T., Mercado-Díaz J. A., Nelsen M. P., Rivas Plata E., Safranek W., Sanders W. B., Schaefer H. P. JR. \& Seavey J. 2011. The lichens of Fakahatchee Strand Preserve State Park, Florida: Proceedings from the 18th Tuckerman Workshop. Bull. Florida Mus. Nat. Hist. 49(4): 127-186.

Lumbsch H. T. 1989. Die holarktischen Vertreter der Flechtengattung Diploschistes (Thelotremataceae). J. Hattori Bot. Lab. 66: 133-196.

Lumbsch H. T. 2002. Diploschistes. In: T. H. Nash III, B. D. RyAn, C. Gries \& F. BungarTz (eds), Lichen flora of the Greater Sonoran Desert Region 1: 173-178. Lichens Unlimited, Arizona State University, Tempe.

Marcano V., Morales Méndez A., Sipman H. J. M. \& CaldeRON L. 1996. A first checklist of the lichen-forming fungi of the Venezuelan Andes. Trop. Bryol. 12: 193-235.

McCarthy P. M. 2013. Checklist of the lichens of Australia and its island territories. Australian Biological Resources Study, Canberra. Version 17 September 2013. http://www. anbg.gov.au/abrs/lichenlist/introduction.html.
Mies B. \& Lumbsch H. T. 1990. Beiträge zur Flechtenflora der Kapverdischen Inseln I. die Gattung Diploschistes. Nova Hedwigia 51(3-4): 483-488.

Nakanishi M., Kashiwadani H., Futagami Y. \& Moon K. H. 2010. Nine species of Graphidaceae (Ostropales, Ascomycota) collected in Siem Reap, Cambodia. J. Jap. Bot. 85(5): 313-321.

Navarro G. \& Ferreira W. 2007. Mapa de vegetación de Bolivia. CD-ROM Interactivo. The Nature Conservancy \& Rumbol, Cochabamba.

Navarro G. \& Maldonado M. 2002. Geografía ecológica de Bolivia: vegetación y ambientes acuáticos. Centro de Ecología Difusíon Simón I. Patiño, Santa Cruz, Bolivia.

Nelsen M. P., LÜCking R., Andrew C. J., Rivas Plata E., Chaves J. L., Cáceres M. E. S. \& Ventura N. 2012. Dismantling Herpothallon: Herpothallon antillarum (Arthoniomycetes: Arthoniaceae) is a member of the genus Diorygma (Lecanoromycetes: Graphidaceae). Bryologist 115(2): 313-321.

Neuwirth G. 2008. Further studies on lichens from Venezuela with new and interesting records. Herzogia 21: 147-156.

Nöske N. M., Mandl N. \& Sipman H. J. M. 2007. Lichens. Checklist Reserva Biológica San Francisco (Prov. ZamoraChinchipe, S. Ecuador). Ecotropical Monographs 4: 101-117.

NyLANDER W. 1859. Lichenes in regionibus exoticis quibusdam vigentes exponit synopticis enumerationibus. Ann. Sci. Nat., Bot. 11: 205-264.

Orange A., James P. W. \& White F. J. 2001. Microchemical methods for the identification of lichens. British Lichen Society, London.

Pant G. \& Upreti D. K. 1993. The lichen genus Diploschistes in India and Nepal. Lichenologist 25(1): 33-50.

REDINGER K. 1935. Die Graphidineen der ersten Regnell‘schen Expedition nach Brasilien 1892-94. II. Graphina und Phaeographina. Ark. Bot. 26A(1): 1-105.

Rivas Plata E. \& LÜCKInG R. 2013. High diversity of Graphidaceae (lichenized Ascomycota: Ostropales) in Amazonian Perú. Fung. Diversity 58(1): 13-32.

Roux C. 2012. Liste des lichens et champignons lichénicoles de France. Bull. Soc. Linn. Provence 16: 3-220.

SAntesson R., Moberg R., Nordin A., Třnsberg T. \& VitiKAINEN O. 2004. Lichen-forming and lichenicolous fungi of Fennoscandia. Museum of Evolution, Uppsala University, Uppsala.

Scholz P. 2001. Rote Liste der Flechten (Lichenes) Thüringens. Naturschutzreport 18: 314-331.

Schubert R. \& Klement O. 1971. Beitrag zur Flechtenflora der Mongolischen Volksrepublik. Feddes Repert. 82: $187-262$. 
Seavey F. \& Seavey J. 2011. The lichen genus Graphis (Graphidaceae) in Everglades National Park (Florida). Bryologist 114(4): 764-784.

Seaward M. R. D. 2010. Census Catalogue of Irish Lichens. National Museums Northern Ireland, Belfast.

Seaward M. R. D., Sipman H. J. M. \& Sohrabi M. 2008. A revised checklist of lichenized, lichenicolous and allied fungi for Iran. Sauteria 15: 459-520.

Sipman H. J. M. 2006. Diversity and biogeography of lichens in Neotropical montane oak forests. Ecological Studies 185: 69-81.

Sipman H. J. M., HeKking W. \& Aguirre-C. J. 2008. Checklist of Lichenized and Lichenicolous Fungi from Colombia. Biblioteca José Jerónimo Triana 20. Instituto de Ciencias Naturales, Facultad de Ciencias, Universidad Nacional de Colombia, Bogotá.

Sipman H. J. M., LÜcking R. Aptroot A. Chaves J. L., Kalb K. \& Umaña Tenorio L. 2012. A first assessment of the
Ticolichen biodiversity inventory in Costa Rica and adjacent areas: the thelotremoid Graphidaceae (Ascomycota: Ostropales). Phytotaxa 55: 1-214.

Staiger B. 2002. Die Flechtenfamilie Graphidaceae. Studien in Richtung einer natürlicheren Gliederung. Biblioth. Lichenol. 85: 1-526.

ThIERs B. 2012. Index Herbariorum: A global directory of public herbaria and associated staff. New York Botanical Garden's Virtual Herbarium. http://sweetgum.nybg.org/ih.

Wirth M. \& Hale M. E. 1978. Morden-Smithsonian Expedition to Dominica: the lichens (Graphidaceae). Smithsonian Contr. Bot. 40: 1-64.

Wolseley P. A., Aguirre-Hudson B. \& McCarthy P. 2002. Catalogue of the lichens of Thailand. Bull. Nat. Hist. Mus. London, Bot. 32(1): 13-59.

YAZICI K. \& Aslan A. 2003. Lichens from the regions of Gümüshane, Erzincan and Bayburt (Turkey). Cryptog. Mycol. 24(2): 287-300.

Received 10 May 2014 